\title{
ON NONISOMORPHIC ROOM SQUARES
}

\author{
J. H. DINITZ AND D. R. STINSON
}

\begin{abstract}
Let $\mathrm{NR}(s)$ denote the number of nonisomorphic Room squares of side $s$. We prove that for $s$ sufficiently large, $\operatorname{NR}(s) \geqslant \exp \left(c s^{2}\right)$ for some absolute constant c. More precisely, $\mathrm{NR}(s) \geqslant .19 \exp \left(.04 s^{2}\right)$ for $s \geqslant 153$ odd; and $\mathrm{NR}(s) \geqslant$ $.19 \exp \left(.09 s^{2}\right)$ for $s \geqslant 1001$ odd.
\end{abstract}

1. Introduction. Let $s$ be a positive odd integer, and let $S$ be a set of size $s+1$. A Room square of side $s$ is an $s$ by $s$ array, $R$, which satisfies the following properties:

$(0)$ each cell of $R$ either is empty or contains an unordered pair of elements ( symbols) of $S$,

(1) every symbol occurs in precisely one cell of each row and column of $R$,

(2) every unordered pair of symbols occurs in precisely one cell of $R$.

Room squares have appeared in the literature as early as 1850 (see Kirkman [5]). They have been studied extensively since the 1960s, and the existence question was solved in 1974 by Mullin and Wallis [8]. We state their result as

THEOREM 1.1. There exists a Room square of side $s$ if and only if $s$ is an odd positive integer other than 3 or 5.

For $i=1,2$, let $R_{i}$ be a Room square of side $s$ based on the symbol set $S_{i}$, and let $\phi: S_{1} \rightarrow S_{2}$ be a bijection. $R_{1}^{\phi}$ is defined to be the Room square based on symbol set $S_{2}$, in which $x$ is replaced by $\phi(x)$ for all $x \in S_{1}$. We say that $R_{1}$ and $R_{2}$ are isomorphic Room squares if there exists some $\phi$ such that $R_{2}$ can be obtained from $R_{\mathrm{l}}^{\phi}$ by permutations of rows and columns. It is clear that isomorphism is an equivalence relation. It is thus natural to ask how many nonisomorphic Room squares exist for each side $s$. We will denote this quantity by $\operatorname{NR}(s)$. We are able to show that $\operatorname{NR}(s) \geqslant \exp \left(c s^{2}\right)$ for some absolute constant $c$, so that clearly $\operatorname{NR}(s)$ grows extremely rapidly.

The transpose of a Room square $R$ is the Room square $R^{T}$ obtained by interchanging the roles of the rows and columns of $R$. Two Room squares $R_{1}$ and $R_{2}$ are equivalent if $R_{1}$ is isomorphic to either of $R_{2}$ or $R_{2}^{T}$ (this also clearly yields an equivalence relation). The number of inequivalent Room squares will be denoted by $\operatorname{IR}(s)$. The following lemma is immediate.

LEMMA 1.2. $\operatorname{IR}(s) \geqslant \frac{1}{2} \mathrm{NR}(s)$

Received by the editors September 30, 1982 and, in revised form, January 26, 1983.

1980 Mathematics Subject Classification. Primary 05B15. 
We note that strict inequality will hold if there exists a Room square of side $s$ which is isomorphic to its transpose. However, our lower bounds for $\mathrm{NR}(s)$ provide bounds for $\operatorname{IR}(s)$ as well.

The question of determining the number of nonisomorphic designs of a given type has been studied for several types of designs: Steiner triple systems (see [15]), Steiner quadruple systems [4], and one-factorizations of complete graphs [1 and 7]. In [6], Lindner establishes that $\operatorname{IR}(s)$ is very large for certain $s$, but our paper provides the first proof that $\mathrm{NR}(s)>1$ for all but a finite number of sides $s$.

The number of inequivalent and nonisomorphic Room squares of side 7 was determined exactly in [3 and 14].

2. A recursive construction. We obtain our lower bounds on $\mathrm{NR}(s)$ by using a recursive construction for Room squares based on group divisible designs. A group divisible design $(\mathrm{GDD})$ is a triple $(X, \mathcal{G}, \mathbb{Q})$ which satisfies:

(0) $X$ is a finite set (elements of which are called points), and $\mathcal{G}$ and $\mathcal{Q}$ are sets of nonempty subsets of $X$ (elements of $\mathcal{G}$ are called groups and elements of $\Theta$ are called blocks),

(1) $\mathcal{G}$ is a partition of $X$,

(2) every block has size at least two, and the groups and blocks together contain every unordered pair of points exactly once.

A transversal design (or $\operatorname{TD}(k, n)$ ) is a GDD with $|X|=k n$, having $k$ groups of size $n$, and $n^{2}$ blocks of size $k$. It is well known that a $\operatorname{TD}(k, n)$ is equivalent to a set of $k-2$ mutually orthogonal Latin squares of order $n$.

Our recursive construction starts with a suitable transversal design, replaces each block by an array called a frame, and then replaces each group by a Room square. Let $X$ be a set, and let $\left\{S_{1}, S_{2}, \ldots, S_{r}\right\}$ be a partition of $X$. An $\left\{S_{1}, S_{2}, \ldots, S_{r}\right\}$-frame is a square array $F$ of size $|X|$, with rows and columns indexed by $X$, which satisfies:

(0) every cell of $F$ either is empty or contains an unordered pair of symbols of $X$,

(1) for every $i, 1 \leqslant i \leqslant r$, the cells of $F\left(s, s^{\prime}\right)$, with $\left\{s, s^{\prime}\right\} \subseteq S_{i}$, are empty (these empty cells are called holes),

(2) each symbol of $X \backslash S_{i}$ occurs in precisely one cell of each row and each column $s \in S_{i}$ of $F$,

(3) the pairs occurring in $F$ are precisely those $\left\{s, s^{\prime}\right\}$ with $\left(s, s^{\prime}\right) \in X^{2} \backslash \cup_{i=1}^{r} S_{i}^{2}$.

Suppose $R$ is a Room square of side $s$ on symbol set $S \cup\{\infty\}(|S|=s)$. Index the rows and columns of $R$ so that $\{\infty, S\}$ occurs in cell $(s, s)$ for each $s \in S$. If the contents of these cells are deleted, an $\{\{s\}: s \in S\}$-frame is formed. Conversely, given such a frame, one can "complete" it to a Room square by filling in the holes (i.e. cells $(s, s)$ ), appropriately.

The type of an $\left\{S_{1}, \ldots, S_{r}\right\}$-frame is the multiset $\left\{\left|S_{i}\right|: 1 \leqslant i \leqslant r\right\}$. We will use the notation $1^{t_{1}} 2^{t_{2}} \cdots$ to describe the type of frame where there are precisely $t_{i} S_{j}$ 's of size $i(i \geqslant 1)$. Thus the above discussion demonstrates that a Room square of side $s$ is equivalent to a frame of type $1^{s}$.

The following two frames are essential ingredients in our recursive construction.

LEMMA 2.1. There exist frames of type $1^{9}$ and $1^{8} 3^{1}$. 
Proof. A frame of type $1^{9}$ is equivalent to a Room square of side 9 , which exists by Theorem 1.1. The frame of type $1^{9}$ in Figure 1 was given by Beaman and Wallis in [2]. The frame of type $1^{8} 3^{1}$ was found by the first author and is presented in Figure 2. Both frames were found by the use of the computer.

Figure 1. A frame of type $1^{9}$

\begin{tabular}{|c|c|c|c|c|c|c|c|c|c|}
\hline & 1 & 2 & 3 & 4 & 5 & 6 & 7 & 8 & 9 \\
\hline 1 & & & 4,9 & 3,7 & 2,8 & & 5,6 & & \\
\hline 2 & 8,9 & & & & & 5,7 & 3,4 & & 1,6 \\
\hline 3 & & 5,8 & & & 6,9 & 2,4 & & 1,7 & \\
\hline 4 & & 3,6 & 7,8 & & & 1,9 & & 2,5 & \\
\hline 5 & & 7,9 & & 1,2 & & 3,8 & & 4,6 & \\
\hline 6 & 4,5 & & & & & & 1,8 & 3,9 & 2,7 \\
\hline 7 & & & 2,6 & 5.9 & 1,3 & & & & 4,8 \\
\hline 8 & 6.7 & 1,4 & & & & & 2,9 & & 3,5 \\
\hline 9 & 2,3 & & 1.5 & 6.8 & 4,7 & & & & \\
\hline
\end{tabular}

Figure 2. A frame of type $1^{8} 3^{1}$

\begin{tabular}{|c|c|c|c|c|c|c|c|c|c|c|c|}
\hline & 1 & 2 & 3 & 4 & 5 & 6 & 7 & 8 & 9 & 10 & 11 \\
\hline 1 & & & & 5,9 & & & 4,8 & 7,11 & & & 6,10 \\
\hline 2 & & & & 7,10 & & & 6,11 & 4,9 & & & 5,8 \\
\hline 3 & & & & & 4,10 & 5,11 & & & 6,8 & 7,9 & \\
\hline 4 & 7,8 & 9,11 & & & & & 1,5 & 2,6 & 3,10 & & \\
\hline 5 & 6,9 & 8,10 & & & & 1,4 & & & & 3,11 & 2,7 \\
\hline 6 & 10,11 & & 8,9 & & 1,7 & & & 3,5 & & 2,4 & \\
\hline 7 & & & & 1,6 & 8,11 & 9,10 & & & 2,5 & & 3,4 \\
\hline 8 & & 5,7 & 4,11 & & 3,6 & & 2,10 & & & & 1,9 \\
\hline 9 & & 4,6 & 5,10 & 2,11 & & 3,7 & & & & 1,8 & \\
\hline 0 & 4,5 & & 6,7 & & & 2,8 & 3,9 & & 1.11 & & \\
\hline 11 & & & & 3,8 & 2,9 & & & 1,10 & 4,7 & 5,6 & \\
\hline
\end{tabular}

Let $(X, \mathcal{G}, \mathcal{Q})$ be a $\operatorname{TD}(9, n)$, where $\mathcal{G}=\left\{G_{i}: 1 \leqslant i \leqslant 9\right\}$, and let $W \subseteq G_{9}$. Denote $t=|W|$. Define a function $S$, with domain $X$, by

$$
S(x)= \begin{cases}x & \text { if } x \in X \backslash W, \\ \left\{x_{i}: 1 \leqslant i \leqslant 3\right\} & \text { if } x \in W .\end{cases}
$$


For every $A \in \mathbb{Q}$, let $F_{A}$ be any $\{S(x): x \in A\}$-frame. (Such a frame is of type $1^{9}$ or $1^{8} 3^{1}$.) Then $F=\cup_{A \in \mathbb{A}^{\prime}} F_{A}$ is a $\left\{\cup_{x \in G,} S(x): 1 \leqslant i \leqslant 9\right\}$-frame of type $n^{8}(n+2 t)^{1}$ by [11, Construction 2.2]. Now, we complete $F$ to a Room square of side $9 n+2 t$. Let $\infty \notin \cup_{x \in X} S(x)$, and place a Room square, on symbol set $\{\infty\} \cup\left(\cup_{x \in G_{i}} S(x)\right)$ in the hole of $F$ induced by $\cup_{x \in G} S(x)$, for $1 \leqslant i \leqslant 9$. The resultant array is a Room square by [12, Theorem 3.1]. We summarize this as

LEMma 2.2. Suppose there is a $\mathrm{TD}(9, n)$, with $n$ odd, and $0 \leqslant t \leqslant n$. If there exist Room squares of sides $n$ and $n+2 t$, then there exists a Room square of side $9 n+2 t$.

Our basic method is as follows. We construct a large number of distinct Room squares on a specified symbol set. We can obtain a naive upper bound on the number of Room squares isomorphic to a given Room square. The quotient of these two quantities provides a lower bound on the number of nonisomorphic Room squares. This is done in the next section.

3: A bound. We first prove that there are many distinct frames of the types $1^{9}$ and $1^{8} 3^{1}$ (on fixed symbol sets).

LEMMA 3.1. There are at least 9! distinct frames of type $1^{9}$, on a fixed symbol set.

Proof. The frame $F_{9}$ of Figure 1 is an $\{\{i\}: 1 \leqslant i \leqslant 9\}$-frame. For II a permutation of $\{1,2, \ldots, 9\}$, let $F_{9}^{\text {II }}$ be the frame defined by $F_{9}^{\text {II }}(\Pi(i), \Pi(j))=\{\Pi(s), \Pi(t)\}$ where $F_{9}(i, j)=\{s, t\}$. It is easily seen that $F_{9}{ }^{I I}$ is also a $\{\{i\}: 1 \leqslant i \leqslant 9\}$-frame. We show that the 9 ! permutations of $\{1,2, \ldots, 9\}$ give rise to distinct frames.

Suppose that $F_{9}^{I I}=F_{9}^{\phi}$. Then, clearly $F_{9}^{I I \phi^{-1}}=F_{9}$. Thus, it suffices to show that $F_{9}^{\Pi} \neq F_{9}$ for all $\Pi$. This can be established with only a moderate amount of case-work.

If $F(i, j)$ is nonempty, then $F(\Pi(i), \Pi(j))$ must be nonempty. Thus, there are only 36 possibilities for $\{\Pi(i), \Pi(j)\}$, determined by the filled cells of $F_{9}$. We let $i=1, j=3$, and consider each case.

All cases are handled similarly. We give an example. Suppose $(\Pi(1), \Pi(3))=(2,7)$. Then $\{\Pi(4), \Pi(9)\}=\{3,4\}=F_{9}(2,7)$. If $\Pi(4)=4$ and $\Pi(9)=3$, then $F(\Pi(4), \Pi(9))=F(4,3)$ must be empty. It is not, so we have a contradiction. Thus, suppose $\Pi(4)=3$ and $\Pi(9)=4$. Then $\{3, \Pi(7)\}=\{\Pi(4), \Pi(7)\}=F(\Pi(9), \Pi(5))$ $=F(4, \Pi(5))$. Since $F(4,2)=\{3,6\}$, we have $\Pi(5)=2$ and $\Pi(7)=6$. Then $F(\Pi(4), \Pi(7))=F(3,6)$ must be empty. It is not, so we have a contradiction in this case as well.

The other cases are handled similarly.

LEMMA 3.2. There are at least $8 !(3 !)^{3}$ distinct frames of type $8^{1} 3^{1}$, on a fixed symbol set.

Proof. We start with the $\{\{1,2,3\},\{i\}: 4 \leqslant i \leqslant 11\}$-frame $F_{11}$ presented in Figure 2. Let $\alpha$ and $\beta$ be permutations of $\{1,2,3\}$ and let $\Pi$ be a permutation of $\{1,2, \ldots, 11\}$ such that $\{\Pi(1), \Pi(2), \Pi(3)\}=\{1,2,3\}$. Define the frame $F_{11}^{\alpha, \beta, \Pi}$ as follows:

for $4 \leqslant i, j \leqslant 11, F_{11}^{\alpha, \beta, \Pi}(\Pi(i), \Pi(j))=\{\Pi(s), \Pi(t)\}$;

for $1 \leqslant i \leqslant 3,4 \leqslant j \leqslant 11, F_{11}^{\alpha, \beta, \Pi}(\alpha(i), \Pi(j))=\{\Pi(s), \Pi(t)\}$; 
and for $4<i<11,1<j<3, F_{11}^{\alpha, \beta \Pi}(\Pi(i), \beta(j))=\{\pi(s), \Pi(t)\}$, where, in each case $F_{11}(i, j)=\{s, t\}$. It is easily shown that each $F_{11}^{\alpha, \beta, \Pi}$ thus constructed is a $\{\{1,2,3\},\{i\}: 4 \leqslant i \leqslant 11\}$-frame. We assert that the $(3 !)^{3} 8$ ! frames thus produced are distinct.

Again, it suffices to show that $F_{11}^{\alpha, \beta, \Pi} \neq F_{11}$ for any $\alpha, \beta, \Pi$. Consider $F_{11}(7,5)=$ $\{8,11\}$. Then $F_{11}^{\alpha, \beta, \Pi}(\Pi(7), \Pi(5))=\{(8), \Pi(11)\}$. Now $\Pi(5), \Pi(7), \Pi(8), \Pi(11) \in$ $\{4,5, \ldots, 11\}$ so we see from $F_{11}$ that $\{\Pi(8), \Pi(11)\}=\{8,11\},\{9,10\},\{4,7\}$ or $\{5,6\}$. These four cases are easily disposed of, as in Lemma 3.1, proving the result.

TheOREM 3.3. Suppose there exists a $\mathrm{TD}(9, n)$, with $n$ odd and $0 \leqslant t \leqslant n$. Then there are at least

$$
(9 !)^{(n-t) n} \cdot\left(8 !(3 !)^{3}\right)^{n t} \cdot\left(2(n !)^{2}\right)^{8} \cdot 2((n+2 t) !)^{2}
$$

distinct Room squares of side $9 n+2 t$, on a fixed symbol set.

ProOF. We apply Lemma 2.2. In the construction of the Room square of side $9 n+2 t$, there are $(n-t) n$ blocks which miss $W$, each of which correspond to a frame of type $1^{9}$. The remaining $t n$ blocks correspond to a frame of type $1^{8} 3^{1}$. We thus obtain $(9 !)^{(n-t) n}\left(8 !(3 !)^{3}\right)^{t n}$ distinct frames of type $n^{8}(n+2 t)^{1}$.

Now we fill in the holes of this frame. Let $R$ be any Room squares of side $n$, by permuting rows and columns of $R$, and transposing (i.e. interchanging the function of rows and columns), we can obtain $2(n !)^{2}$ distinct Room squares of side $n$. We fill in eight Room squares of side $n$ and one of side $n+2 t$; so each frame can be completed in at least $\left(2(n !)^{2}\right)^{8} 2((n+2 t) !)^{2}$ ways.

It is easily seen that all the Room squares obtained by this construction are distinct.

COROllary 3.4. Suppose there exists $a \operatorname{TD}(9, n)$ and $9 n \leqslant s \leqslant 11$, where $n$ and $s$ are odd. Then

$$
\mathrm{NR}(s) \geqslant \frac{(9 !)^{n^{2}} 2^{9}(n !)^{18}}{((11 n) !)^{2}(11 n+1) !}
$$

Proof. First, write $s=9 n+2 t$, where $0 \leqslant t \leqslant n$. We divide the lower bound for the number of distinct Room squares of side $s$ obtained in Theorem 3.3 by the number of possible distinct Room squares isomorphic to a given Room square of side $s$. This number can be at most $((9 n+2 t) !)^{2}(9 n+2 t+1)$ ! (allowing perumtations of rows, columns and symbols). Thus,

$$
\begin{aligned}
\mathrm{NR}(s) & =\mathrm{NR}(9 n+2 t) \geqslant \frac{(9 !)^{(n-t) n}\left(8 !(3 !)^{3}\right)^{t n}\left(2(n !)^{2}\right)^{8} 2((n+2 t) !)^{2}}{((9 n+2 t) !)^{2}(9 n+2 t+1) !} \\
& >\frac{(9 !)^{n^{2}} 2^{9}(n !)^{18}}{((11 n) !)^{2}(11 n+1) !} .
\end{aligned}
$$

We examine the behavior of the above quantity with the following version of Stirlings's formula (see [9]). 
LEMMA 3.5. $n !=(2 \pi n)^{1 / 2}(n / e)^{n} e^{\alpha_{n}}$ where $1 /(12 n+1)<\alpha_{n}<1 / 12 n$.

THEOREM 3.6. Suppose there is a $\mathrm{TD}(9, n)$ and $9 n \leqslant s \leqslant 11 n$ where $n$ and $s$ are odd. Then

$$
\mathrm{NR}(s)>c \cdot \exp \left(n^{2} \ln (9 !)-15 n \ln (n)-(33 \ln 11-15) n+\frac{13}{2} \ln (n)\right),
$$

where $c=\left(2^{29} \pi^{15} /\left(9 \cdot 11^{3}\right)\right)^{1 / 2}$.

Proof. From Corollary 3.4, we have

$$
\begin{aligned}
\mathrm{NR}(s) & >\frac{2^{9} \cdot(9 !) n^{2} \cdot(n !)^{18}}{((11 n) !)^{3}(11 n+1)} \\
& \geqslant \frac{2^{9}(9 !)^{n^{2}}(2 \pi n)^{9}(n / e)^{18 n} e^{18 /(12 n+1)}}{(22 \pi n)^{3 / 2}(11 n / e)^{33 n} e^{3 /(12 \cdot 11 n)}(11 n+1)} \quad(\text { Lemma 3.5) } \\
& >\frac{2^{9}(9 !)^{n^{2}}(2 \pi n)^{9}(n / e)^{18 n}}{(22 \pi n)^{3 / 2}(1 \ln / e)^{33 n} \cdot 12 n} \\
& =\left(\frac{2^{29} \cdot \pi^{15}}{9 \cdot 11^{3}}\right)^{1 / 2} \exp \left(n^{2} \ln (9 !)-15 n \ln (n)-(33 \ln 11-15) n+\frac{13}{2} \ln (n)\right) .
\end{aligned}
$$

The above bound is dependent upon the existence of certain transversal designs. The following result is well known.

LEMMA 3.7. Let $n$ have prime power factorization $\pi p_{i}^{\alpha i}$. If $k \leqslant 1+\min \left\{p_{i}^{\alpha{ }^{\prime}}\right\}$, then there exists a $\operatorname{TD}(k, n)$.

One can then prove the following by use of the above lemma and a simple arithmetic argument (see [13]).

COROllaRy 3.8. If $s \geqslant 153$, then there is a positive integer $n$, with $9 n \leqslant s \leqslant 11 n$, such that a $\mathrm{TD}(9, n)$ exists.

Elementary calculus shows that the bound of Corollary 3.6 is an increasing function of $n$ (for $n \geqslant 9$, for example). Thus we have

THEOREM 3.9. For $s \geqslant 153$ odd,

$$
\mathrm{NR}(s)>c \cdot \exp \left(\frac{s^{2}}{121} \ln (9 !)-\frac{15}{11} s \ln (s)+\left(\frac{15}{11}-\frac{18}{11} \ln (11)\right) s+\frac{13}{2} \ln (s)\right),
$$

where $c=\left(2^{29} \pi^{15} /\left(9 \cdot 11^{16}\right)\right)^{1 / 2}>.19$.

Corollary 3.10. (1) For $s \geqslant 153$, sodd, NR(s)>.19 $e^{.04 s^{2}}>1$.

(2) For $s \geqslant 1001, s$ odd, $\mathrm{NR}(s)>.19 e^{.09 s^{2}}$.

(3) $\mathrm{NR}(1001)>10^{40850}$.

4. Summary and remarks. We have shown that the number of nonisomorphic (and inequivalent) Room squares grows extremely rapidly. The techniques in this paper are quite general. A similar argument involving group-divisible designs could be used, for example, to show that the number of nonisomorphic $(v, k, 1)$ balanced incomplete block designs approaches infinity as $v$ approaches infinity. 


\section{REFERENCES}

1. B. A. Anderson, M. M. Barge and D. Morse, A recursive construction of asymmetric 1-factorizations, Aequationes Math. 15 (1977), 201-211.

2. I. R. Beaman and W. D. Wallis, A skew Room square of side 9, Utilitas Math. 8 (1975), 382.

3. K. B. Gross, Equivalence of Room designs. I and II, J. Combin. Theory Ser. A 16 (1974), 264-265; 17 (1974), 299-316.

4. A. Hartman, Counting quadruple systems, Congr. Numer. 33 (1981), 45-54.

5. T. P. Kirkman, Note on an unanswered prize question, Cambridge and Dublin Math. J. 5 (1850), $255-262$.

6. C. C. Lindner, An algebraic construction for Room squares, SIAM J. Appl. Math. 22 (1972), 574-579.

7. C. C. Lindner, E. Mendelsohn and A. Rosa, On the number of 1-factorizations of the complete graph, J. Combin. Theory Ser. B 20 (1976), 265-282.

8. R. C. Mullin and W. D. Wallis, The existence of Room squares, Aequationes Math. 13 (1975), 1-7.

9. H. Robbins, Stirlings formula, Amer. Math. Monthly 62 (1955), 26-29.

10. A. Rosa, Room squares generalized, Ann. Discrete Math. 8 (1980), 43-57.

11. D. R. Stinson, Some constructions for frames, Room squares, and subsquares, Ars. Combin. 12 (1981), 229-268.

12. Some results concerning frames, Room squares, and subsquares, J. Austral. Math. Soc. Ser. A 31 (1981); 376-384.

13. _ The existence of Howell designs of odd side, J. Combin. Theory Ser. A 32 (1982), 53-65.

14. W. D. Wallis, A. P. Street and J. S. Wallis, Combinatorics: Room squares, sum-free sets, Hadamard matrices, Lecture Notes in Math., Springer-Verlag, Berlin, 1972.

15. R. M. Wilson, Nonisomorphic Steiner triple systems, Math. Z. 135 (1974), 303-313.

Department of Mathematics, University of Vermont, Burlington, Vermont 05405

Department of Computer Science, University of Manitoba, Winnepeg, Manitoba, R3T 2N2 CANADA 